\title{
RESISTÊNCIA DE PAINÉIS COMPENSADOS DE Pinus Taeda TRATADOS COM PRESERVANTES AO ATAQUE DE FUNGOS XILÓFAGOS
}

\author{
Rafael Farinassi Mendes ${ }^{1 *}$, Geraldo Bortoletto Júnior ${ }^{2}$, Alencar Garlet³ ${ }^{3}$ Jackson Marcelo Vidal $^{2}$, Natalie Ferreira de \\ Almeida ${ }^{2}$, Ivaldo Pontes Jankowsky ${ }^{2}$
}

*Autor para correspondência: rafaelfarinassi@gmail.com

RESUMO: O trabalho teve como objetivo verificar o efeito de diferentes produtos preservantes e de diferentes retenções do produto sobre a resistência de painéis compensados de Pinus taeda ao ataque de fungos xilófagos. O delineamento experimental se constituiu de seis tratamentos, sendo dois tipos de produtos preservantes (CCA e CCB), duas retenções $\left(4,0\right.$ e $\left.6,5 \mathrm{~kg} / \mathrm{m}^{3}\right)$, um tratamento apenas com água, o qual teve como objetivo avaliar o efeito apenas do processo de aplicação dos preservantes, e um tratamento testemunha (sem tratamento). Os painéis compensados utilizados foram produzidos com a madeira de Pinus taeda, cinco lâminas de 3 mm de espessura e com o adesivo fenol-formaldeído na gramatura de $420 \mathrm{~g} / \mathrm{m}^{2}$ (linha dupla). Para a avaliação da resistência a biodegradação dos painéis compensados foi utilizada a metodologia proposta pela norma ASTM D2017(2005), sendo avaliados os fungos Trametes versicolor e Gloeophyllum trabeum. Na avaliação da resistência a biodeterioração dos painéis ao ataque do fungo Trametes versicolor não foi observado efeito significativo do tipo de produto e da retenção aplicada. Na avaliação quanto à resistência ao ataque do fungo Gloeophyllum trabeum, o produto CCA aplicado na retenção de $4,0 \mathrm{~kg} / \mathrm{cm}^{3}$ proporcionou maior resistência a biodeterioração que o produto CCB também na mesma retenção, e na avaliação das duas retenções do produto CCB observou-se que o aumento da retenção para $6,5 \mathrm{~kg} / \mathrm{m}^{3}$ resultou em melhora significativa na resistência a biodegradação dos painéis.

Palavras-chave: Biodeterioração, CCA e CCB, retenção.

\section{RESISTANCE OF Pinus taeda PLYWOOD TREATED WITH PRESERVATIVESTO ATTACK BY DECAY FUNGI}

\begin{abstract}
The study aimed to investigate the effect of different preservatives products and retentions on the resistance of Pinus taeda plywood to attack by decay fungi. The experiment consisted of six treatments, two types of products preservatives (CCA and CCB), two retentions (4.0 and $6.5 \mathrm{~kg} / \mathrm{m}^{3}$ ), one treatment with water only, which aimed to evaluate the effect only the process of preservatives application, and a control (no treatment). The plywood was produced with Pinus taeda wood, five veneers of $3 \mathrm{~mm}$ thickness and phenol-formaldehyde adhesive at a weight of $420 \mathrm{~g} / \mathrm{m}^{2}$ (double line). To evaluate the resistance to biodegradation of plywood was used the methodology proposed by ASTM D2017 (2005), and evaluated the fungi Trametes versicolor and Gloeophyllum trabeum. In the resistance evaluation of the panels biodeterioration to attack the fungus Trametes versicolor was no significant effect of product type and retention. The resistance evaluation to attack by fungus Gloeophyllum trabeum, CCA applied in retention of $4.0 \mathrm{~kg} / \mathrm{cm}^{3}$ provided greater resistance to biodeterioration than $C C B$ in the same retention, and in the evaluation of two retentions of $C C B$ there was observed that retention increase to $6.5 \mathrm{~kg} / \mathrm{m}^{3}$ resulted in significant improvement in the panels resistance to biodegradation..
\end{abstract}

Key words: Biodeterioration, CCA and CCB, retention.

\section{INTRODUÇÃO}

Os painéis compensados são produtos reconhecidos pelas suas excelentes propriedades físicas e mecânicas, sendo usados em grande escala na construção civil. Sua durabilidade natural é determinada, principalmente, pela espécie utilizada na sua produção (TASCIOGLU e TSUNODA, 2010a). Contudo, o uso de espécies mais duráveis a biodegradação podem vir a afetar vários parâmetros de produção, dentre os quais: a formulação e

\footnotetext{
${ }^{1}$ Universidade Federal de Lavras - Lavras, Minas Gerais, Brasil

${ }^{2}$ Universidade de São Paulo - Piracicaba, São Paulo, Brasil

${ }^{3}$ Serviço Florestal Brasileiro - Brasília, Distrito Federal, Brasil
}

penetração adequada dos adesivos, a quantidade utilizada de adesivo e o ciclo de prensagem; haja visto que normalmente apresentam maior porcentagem de extrativos e maior densidade (ALMEIDA et al., 2012), fazendo com que espécies de madeira menos duráveis sejam cada vez mais utilizadas na produção de painéis compensados (VAN ACKER e STEVENS, 1993).

Como os painéis compensados podem estar expostos a condições desfavoráveis, tais como ineficiente ventilação e elevada umidade, vindo a sofrer o processo

Cerne, Lavras, v. 20, n. 1, p. 105-112, jan./mar. 2014 
de deterioração por fungos e cupins (FOJUTOWSKI e KROPACZ, 2008; BULCKE et al., 2009), cada vez mais busca-se o tratamento desses painéis com algum tipo de produto preservativo, podendo ser inseridos por meio da linha de cola ou aplicados por meio de vácuo-pressão, sendo assim capazes de prolongar sua vida útil (WANG e HENNINGSSON, 1989).

Portanto, atualmente, a preservação não deve ser considerada como custo adicional, mas um investimento indispensável. Proporcionando à madeira condições de durabilidade para competir no mercado consumidor com os demais materiais de construção e, consequentemente, poder gerar maior economia na utilização dos recursos florestais e incentivar o emprego do material.

Tsunoda (2001) ao avaliar o tratamento com vaporização de boro em painéis compensados, produzidos com a madeira Cryptomeria japônica e adesivo fenol-formaldeído, observaram perda de massa causada pelos fungos Trametes versicolor e Fomitopsis palustris da ordem de 15,5 e $0,6 \%$, respectivamente, enquanto que o valor obtido para a madeira sem tratamento, também para os dois tipos de fungos, foram de 52,9 e $59,7 \%$, respectivamente.

Loh et al. (2001) ao avaliar o tratamento de lâminas de madeira com óleo extraído de palmeiras, para posterior produção de painéis compensados com adesivo ureiaformaldeído na gramatura de $200 \mathrm{~g} / \mathrm{m}^{2}$ (linha simples), encontraram valores de perda de massa, causadas pelo fungo Coptotermes curvignathus, da ordem de $13 \%$. Para os painéis sem tratamento, o valor médio obtido para perda de massa foi de $34 \%$.

Em geral, os preservativos hidrossolúveis CCA (Arseniato de cobre cromatado) e CCB (Borato de cobre cromatado) são os mais utilizados no tratamento de madeiras maciças, sendo que quando aplicados a madeira, o produto reage com os constituintes químicos da mesma, tornando-se insolúvel e eficaz contra a ação de agentes biodegradadores (GALVÃO et al., 2004).

Além do tipo de produto aplicado, Mazela (2000) afirma que a resistência da madeira tratada aos agentes biológicos é, também, bastante influenciada pela retenção de aplicação do produto preservante. O que torna necessário para cada tipo de produto à base de madeira e para cada tipo de produto preservante, a busca de uma otimização da quantidade a ser aplicada, visto que o tratamento preservante pode vir a causar aumento significativo nos custos de comercialização dos painéis.

Cerne, Lavras, v. 20, n. 1, p. 105-112, jan./mar. 2014
Neste contexto, o presente trabalho teve como objetivo verificar o efeito de dois dos principais produtos preservantes empregados atualmente, sob duas diferentes retenções, sobre a resistência dos painéis compensados ao ataque de fungos xilófagos.

\section{MATERIAL E MÉTODOS}

\subsection{Material de estudo}

Foram analisados corpos-de-prova retirados de painéis compensados produzidos com a madeira de Pinus taeda aos 35 anos de idade e com densidade básica média de $0,473 \mathrm{~g} / \mathrm{cm}^{3}$. Outros parâmetros utilizados para a produção dos painéis foram a utilização de cinco lâminas de madeira com espessura de $3 \mathrm{~mm}$, adesivo fenolformaldeído na gramatura de $420 \mathrm{~g} / \mathrm{m}^{2}$ (linha dupla) e formulação do adesivo, em partes por peso, para a aplicação de: adesivo $\mathrm{FF}=100$; farinha de trigo $=10$; farinha de casca de $\operatorname{coco}=5$; e água $=10$. Sendo realizado, após a aplicação do adesivo nas lâminas, o tempo de assemblagem de 15 minutos e a execução do ciclo de prensagem com os seguintes parâmetros: temperatura de $150^{\circ} \mathrm{C}$; tempo e pressão de prensagem de 15 minutos e $11 \mathrm{kgf} / \mathrm{cm}^{2}$, respectivamente. Para cada um dos tratamentos foram produzidos três painéis.

Após a produção dos painéis estes sofreram diferentes tipos de tratamentos preservantes, conforme descrito na Tabela 1 .

Tabela 1- Tratamentos aplicados aos painéis compensados

Table 1 - Treatments applied to plywood

\begin{tabular}{ccc}
\hline Tratamento & Produto & Retenção $\left(\mathrm{kg} / \mathrm{m}^{3}\right)$ \\
\hline 1 & CCA & 4,0 \\
2 & CCA & 6,6 \\
3 & CCB & 4,0 \\
4 & CCB & 6,5 \\
5 & Água & - \\
$6^{*}$ & - & - \\
\hline
\end{tabular}

* Testemunha

Neste delineamento foi avaliado dois tipos de produtos preservantes (CCA - Arseniato de cobre cromatado e CCB - Borato de cobre cromatado) em duas retenções $\left(4,0\right.$ e $\left.6,5 \mathrm{~kg} / \mathrm{m}^{3}\right)$. Além disso, realizou-se um tratamento testemunha (sem tratamento preservante) e também um tratamento apenas com água, o qual visou 
avaliar uma possível modificação advinda do efeito do processo de tratamento preservante utilizado.

O tratamento preservante dos painéis foi efetuado pelo método de vácuo-pressão. Após as amostras inseridas no interior de uma autoclave, foram realizadas as seguintes etapas: 1) Vácuo inicial de $570 \mathrm{mmHg}$, por um período de 30 minutos; 2) Aplicação do produto sob pressão de 11,0 $\mathrm{kgf} / \mathrm{cm}^{2}$, por um período de 60 minutos; e 3) Vácuo final para retirada do excesso do produto de $570 \mathrm{mmHg}$, por um período de 10 minutos. Esse procedimento foi utilizado com os dois tipos de produtos (CCA e CCB) e também nos painéis com tratamento apenas com água.

\subsection{Preparação dos corpos-de-prova}

O ensaio de apodrecimento acelerado foi realizado na Área de Biodegradação e Preservação da Madeira, do Laboratório de Produtos Florestais (LPF) / Serviço Florestal Brasileiro, em Brasília, DF. Utilizou-se a metodologia proposta pela norma ASTM D2017(2005).

Para tanto, os corpos-de-prova foram preparados nas dimensões de $25 \times 25 \mathrm{~mm}$ X espessura do painel. Foram ensaiados 12 corpos-de-prova (4 por painel) por tratamento para cada fungo.

Todos os corpos-de-prova foram acondicionados em estufa com circulação forçada de ar a $50^{\circ} \mathrm{C}$, até atingir massa constante. Posteriormente, foram pesados em balança analítica, com resolução de $0,0001 \mathrm{~g}$, e o resultado registrado como Mi (massa inicial). Após a pesagem inicial os corpos-de-prova foram esterilizados em autoclave à $121^{\circ} \mathrm{C}$, por 30 minutos.

\subsection{Fungos xilófagos}

Foram empregadas duas espécies de fungos xilófagos provenientes da coleção do LPF: Trametes versicolor (Linnaeus ex Fries) Pilat, espécie causadora de podridão branca, predominante em madeira de folhosas; e Gloeophyllum trabeum (Persoon. ex Fries.) Murr., espécie causadora de podridão parda.

Os fungos foram repicados em meio de cultura malte líquido à $3 \%$, e levados à incubadora $\left(26 \pm 1{ }^{\circ} \mathrm{C}\right.$ e $70 \pm 5 \%$ de umidade relativa), até o micélio recobrir totalmente a superfície do meio (três semanas). No momento da inoculação o micélio foi fragmentado com liquidificador doméstico e, em seguida, pipetado para os frascos de ensaio. Todas as operações de manipulação de fungos (repicagem, inoculação, fragmentação do micélio, introdução de corpos-de-prova nos frascos de ensaio) foram realizadas sob condições assépticas, no interior de capela de fluxo laminar.

\subsection{Ensaio de apodrecimento}

Foram utilizados frascos de vidro transparente, com boca larga, tampa rosqueável e capacidade de $250 \mathrm{~mL}$, contendo $100 \mathrm{~g}$ de solo (horizonte B de solo podzólico vermelho-amarelo), livre de matéria orgânica. O solo recebeu adição prévia de calcário, para elevar o $\mathrm{pH}$ a 6,0 . A umidade do solo foi ajustada para $130 \%$ da capacidade de campo.

Em cada frasco foi colocada uma placa suporte de 33 X 29 X $3 \mathrm{~mm}$ para o desenvolvimento inicial do fungo. Foram utilizadas placas suporte de pinus (Pinus $s p$.), para o cultivo de Gloeophyllum trabeum; e placas de embaúba (Cecropia sp.) para o cultivo de Trametes versicolor. Os frascos foram autoclavados a $121^{\circ} \mathrm{C}$ por 45 minutos. Cada frasco foi inoculado com $1 \mathrm{ml}$ de meio de cultura, contendo o micélio fragmentado, depositado diretamente sobre a placa suporte.

Após a inoculação os frascos foram levados à incubadora com temperatura de $26 \pm 1^{\circ} \mathrm{C}$ e $70 \pm 5 \%$ de umidade relativa, até o micélio cobrir totalmente a placa suporte (2 semanas). Após a colonização das placas suporte, colocou-se um corpo-de-prova em cada frasco de ensaio. As amostras dos painéis permaneceram em contato com os fungos por 12 semanas na câmara climática em condições já descritas.

Encerrado o período de apodrecimento, os corpos-de-prova foram retirados dos frascos de ensaio e submetidos a uma limpeza cuidadosa para remoção do excesso de micélio aderido. Posteriormente, foram condicionados em estufa, sob as mesmas condições préensaio. Após atingirem massa constante, foram novamente pesados para determinação da massa final (Mf).

\subsubsection{Blocos de correção}

Para cada tratamento seis corpos-de-prova foram introduzidos em frascos de ensaio não inoculados (sem fungos) e receberam o mesmo tratamento e manipulação dos demais. Os blocos de correção têm o objetivo de determinar a perda de massa resultante da manipulação dos corpos-de-prova, não causada pela ação dos fungos.

Cerne, Lavras, v. 20, n. 1, p. 105-112, jan./mar. 2014 


\subsubsection{Espécies de referência}

Foram utilizados 18 corpos-de-prova $(25$ X 25 X 9 $\mathrm{mm}$ ) de pinus (Pinus sp.) como espécie de referência para o fungo G. trabeum e 18 corpos-de-prova de embaúba (Cecropia sp.) para o fungo T. versicolor. Este modelo permite a retirada de grupos de 6 corpos-de-prova, após 12,14 e 16 semanas de ensaio. $\mathrm{O}$ ensaio foi encerrado quando a perda de massa média da espécie referência foi superior a $50 \%$.

Os corpos-de-prova de referência são indicadores do vigor vegetativo da cultura fúngica utilizada no ensaio e das condições de incubação. Segundo a norma ASTM D-2017:05, o teste é considerado válido somente quando o fungo provoca perda de massa de $50 \%$ sobre a espécie de referência, num período de 12 até 16 semanas.

A seleção das espécies de referência levou em consideração a existência de dados de vários ensaios realizados no LPF, que comprovam serem estas espécies susceptíveis aos fungos utilizados.

\subsection{Classificação de resistência e análise estatística}

A partir dos valores de massa inicial e final foi calculada a perda de massa dos corpos de prova conforme a equação 1 :

$\operatorname{Pmt}(\%)=((\mathrm{Mi}-\mathrm{Mf}) /(\mathrm{Mi})) \times 100$

Onde: Pmt $=$ perda de massa total

$\mathrm{Mi}=$ massa inicial

$\mathrm{Mf}=$ peso final

Com os valores de perda de massa total e perda de massa dos blocos de correção, foi calculada a perda de massa corrigida, conforme a equação 2 .

Pmc $(\%)=$ Pmt - Pmbc

Onde: Pmc $=$ Perda de massa corrigida

$\mathrm{Pmt}=$ Média de perda de massa total

Pmbc $=$ Média de perda de massa dos blocos de correção do tratamento

Com base na média da perda de massa corrigida, os tratamentos foram classificados quanto à resistência ao ataque dos fungos, segundo os critérios estabelecidos na norma ASTM D 2017 (ASTM, 2005) e apresentados na Tabela 2.
Tabela 2 - Classes de resistência ao apodrecimento segundo a norma ASTM D 2017 (ASTM, 2005).

Table 2 - Decay resistance classes according to ASTM D 2017 (ASTM, 2005).

\begin{tabular}{ccc}
\hline Classe de Resistência & $\begin{array}{c}\text { Perda de Massa } \\
(\%)\end{array}$ & $\begin{array}{c}\text { Massa Residual } \\
(\%)\end{array}$ \\
\hline Alta Resistência (AR) & $0-10$ & $90-100$ \\
Resistente (R) & $11-24$ & $76-89$ \\
Resistência Moderada (RM) & $25-44$ & $56-75$ \\
Não-Resistente (NR) & $>45$ & $>55$ \\
\hline
\end{tabular}

A análise estatística dos dados foi realizada separadamente para cada um dos tipos de fungos, sendo considerado um experimento em delineamento inteiramente casualizado, em que os tratamentos estavam arranjados em um esquema fatorial $2 \times 2$ (dois produtos - CCA e CCB; duas retenções - 4,0 e $\left.6,5 \mathrm{~kg} / \mathrm{m}^{3}\right)$, e ainda com dois tratamentos adicionais, um tratamento com água e o outro como testemunha (sem tratamento preservante). Para a comparação entre os painéis que passaram por algum tratamento e os painéis testemunha, foi realizado o teste de Dunnett a 5\% de probabilidade. Enquanto que o teste Tukey, também a $5 \%$ de probabilidade, foi realizado para as propriedades que apresentaram interação significativa entre os tipos de produtos preservantes e as diferentes retenções.

\section{RESULTADOS E DISCUSSÃO}

A perda de massa observada nas madeiras de referência foi de $50,76 \%$ na madeira de embaúba, causada pelo fungo Trametes versicolor, e de 53,33\% na madeira de pinus, causada pelo fungo Gloeophyllum trabeum. Estes valores foram observados após 12 semanas de ensaio e são superiores ao valor mínimo de 50\% estabelecido pela norma ASTM D 2017 (ASTM, 2005). Assim, este ensaio foi encerrado após 12 semanas de exposição dos corposde-prova aos fungos apodrecedores.

Estes dados atestam que as condições gerais de ensaios foram favoráveis ao crescimento dos fungos, e que as culturas empregadas estavam em pleno vigor vegetativo. Portanto, os resultados são válidos de acordo com os critérios da norma.

Os valores médios de perda de massa observados para os painéis compensados sob o ataque dos fungos Trametes versicolor e Gloeophyllum trabeum estão apresentados na Tabela 3.

Cerne, Lavras, v. 20, n. 1, p. 105-112, jan./mar. 2014 
Tabela 3 - Valores médios de perda de massa dos painéis compensados

Table 3 - Average values of plywood mass loss

\begin{tabular}{ccccc}
\hline & \multicolumn{4}{c}{ Perda de massa (\%) } \\
\cline { 2 - 5 } Tratamentos & $\begin{array}{c}\text { Trametes } \\
\text { versicolor }\end{array}$ & $\begin{array}{c}\text { Classe de } \\
\text { resistência }\end{array}$ & $\begin{array}{c}\text { Gloeophyl- } \\
\text { lum } \\
\text { trabeum }\end{array}$ & $\begin{array}{c}\text { Classe de } \\
\text { resistência }\end{array}$ \\
\hline $\begin{array}{c}\mathrm{CCA} \\
\left(4,0 \mathrm{~kg} / \mathrm{m}^{3}\right)\end{array}$ & $0,31(0,17)^{*}$ & $\mathrm{AR}$ & $0,09(0,07)^{*}$ & $\mathrm{AR}$ \\
$\begin{array}{c}\mathrm{CCA} \\
\left(6,5 \mathrm{~kg} / \mathrm{m}^{3}\right)\end{array}$ & $0,16(0,12)^{*}$ & $\mathrm{AR}$ & $0,04(0,03)^{*}$ & $\mathrm{AR}$ \\
$\begin{array}{c}\mathrm{CCB} \\
\left(4,0 \mathrm{~kg} / \mathrm{m}^{3}\right)\end{array}$ & $0,33(0,12)^{*}$ & $\mathrm{AR}$ & $2,29(1,38)^{*}$ & $\mathrm{AR}$ \\
$\begin{array}{c}\mathrm{CCB} \\
\left(6,5 \mathrm{~kg} / \mathrm{m}^{3}\right)\end{array}$ & $0,47(0,21)^{*}$ & $\mathrm{AR}$ & $0,16(0,10)^{*}$ & $\mathrm{AR}$ \\
$\begin{array}{c}\text { Agua } \\
\text { Testemunha }\end{array}$ & $11,38(4,56)^{\mathrm{ns}}$ & $\mathrm{R}$ & $31,04(5,05)^{*}$ & $\mathrm{RM}$ \\
\hline $\begin{array}{c}\text { Pinus } \\
\text { (referência) }\end{array}$ & $11,52(4,23)$ & $\mathrm{R}$ & $35,34(3,70)$ & $\mathrm{RM}$ \\
$\begin{array}{c}\text { Embaúba } \\
\text { (referência) }\end{array}$ & $50,76(3,63)$ & $\mathrm{NR}$ & - & $\mathrm{NR}$ \\
\hline
\end{tabular}

* Difere estatisticamente pelo Teste de Dunnett $(\alpha=0,05)$ do tratamento testemunha; ${ }^{\text {ns }}$ Não difere estatisticamente pelo teste de Dunnett $(\alpha=0,05)$ do tratamento testemunha. $A R=$ Alta resistência; $\mathrm{R}=$ resistente; $\mathrm{RM}=$ resistência modera; $\mathrm{NR}=$ não resistente, segundo ASTM D 2017 (ASTM, 2005). Valores dentro dos parênteses são os desvios padrões.

Pela análise do teste de Dunnett ( $\alpha=0,05)$, observouse que as aplicações dos dois tipos de produtos (CCA e CCB) nas diferentes retenções $\left(4,0\right.$ e $\left.6,5 \mathrm{~kg} / \mathrm{m}^{3}\right)$ causaram melhora significativa em relação aos painéis testemunha quanto ao ataque dos fungos Trametes versicolor e Gloeophyllum trabeum.

Quando comparado os painéis testemunhas com os painéis tratados apenas com água, foi possível verificar diferença significativa quanto à perda de massa apenas para o fungo Gloeophyllum trabeum. Como os painéis que sofreram o tratamento com água obtiveram os menores valores médios de perda de massa, pode-se afirmar que uma pequena parte do aumento da resistência dos painéis compensados a biodegradação a este fungo está associada ao método de aplicação dos produtos.

Tascioglu e Tsunoda (2010b) avaliando a resistência de diferentes tipos de painéis ao ataque de fungos do tipo Trametes versicolor também observou diminuição da perda de massa de painéis compensados quando os painéis foram tratados em autoclave apenas com água. Possivelmente tal fato pode estar associado a alguma mudança causada na estrutura anatômica das lâminas após os painéis passarem pelo processo de vácuo-pressão.

Ainda de acordo com os resultados da análise de variância, não foi observada interação entre os tipos de produtos preservantes e as diferentes retenções para a perda de massa causada pelo fungo Trametes versicolor, assim como também não foi verificado efeito para os dois fatores analisados de forma separada (Tabela 4).

$\mathrm{Na}$ análise do fungo Gloeophyllum trabeum foi observada interação significativa entre o tipo de produto

Tabela 4 - Perda de massa causada pelo fungo Trametes versicolor

Table 4 - Mass loss caused by the Trametes versicolor fungus

\begin{tabular}{cccc}
\hline \multicolumn{4}{c}{ Perda de massa (\%) } \\
\hline \multicolumn{3}{c}{ Produto } & \multicolumn{2}{c}{ Retenção } \\
\hline CCA & $0,24 \mathrm{a}$ & 4,0 & $0,65 \mathrm{a}$ \\
$\mathrm{CCB}$ & $0,40 \mathrm{a}$ & 6,5 & $0,63 \mathrm{a}$ \\
\hline
\end{tabular}

Médias seguidas de mesma letra na coluna não diferem estatisticamente pelo teste de Tukey $(\alpha=0,05)$.

e a retenção aplicada. Os resultados apresentados para a análise de desdobramento (Tabela 5) indicaram que houve efeito dos dois tipos de produtos (CCA e CCB) na retenção de $4,0 \mathrm{~kg} / \mathrm{m}^{3}$, sendo o produto CCA o que apresentou os menores valores médios para a perda de massa. Também foi observado o efeito da retenção aplicada quando analisado o produto $\mathrm{CCB}$, com a menor retenção a que apresentou maior perda de massa dos painéis compensados.

Contudo, apesar da diferenciação estatística entre os tipos de produtos e retenções de aplicações dos

Tabela 5 - Desdobramento da interação para o fungo Gloeophyllum trabeum

Table 5 - Interaction deployment for the Gloeophyllum trabeum fungus

\begin{tabular}{ccc}
\hline \multirow{2}{*}{ Produto } & \multicolumn{2}{c}{ Retenção $\left(\mathrm{kg} / \mathrm{m}^{3}\right)$} \\
\cline { 2 - 3 } & 4,0 & 0,65 \\
\hline CCA & $0,09 \mathrm{aA}$ & $0,04 \mathrm{aA}$ \\
CCB & $2,29 \mathrm{bB}$ & $0,16 \mathrm{aA}$ \\
\hline
\end{tabular}

Médias seguidas de mesma letra não diferem estatisticamente pelo teste de Tukey $(\alpha=0,05)$. As letras minúsculas referem aos valores das colunas e as letras maiúsculas aos valores das linhas

mesmos, todos tratamentos preservantes foram eficientes contra ambos os fungos testados (Tabela 3). Além disso, os

Cerne, Lavras, v. 20, n. 1, p. 105-112, jan./mar. 2014 
tratamentos em que foram aplicados algum tipo de produto preservante, independente da retenção, foram classificados como altamente resistentes, de acordo com os critérios da norma ASTM D 2017 (ASTM, 2005), para ambos os fungos avaliados.

O tratamento testemunha apresentou perda de massa de $11,52 \%$ para T. versicolor e $35,54 \%$ para G. trabeum. Resultados semelhantes foram apresentados pelo tratamento dos painéis apenas com água, com perda de massa de 11,38\% para T. versicolor e 31,04\% para $G$. trabeum. Ambos os tratamentos foram classificados como resistentes ao fungo de podridão branca e moderadamente resistentes ao fungo de podridão parda.

Tsunoda (2001) ao avaliar o tratamento com vaporização de boro de painéis compensados, produzidos com a madeira Cryptomeria japonica e adesivo fenolformaldeído, observaram perda de massa causada pelos fungos Trametes versicolor da ordem de 15,5\%, podendo ser os painéis classificados como resistentes, conforme especificações da norma ASTM D2017 (2005).

Vaz (2013) ao avaliar o tratamento térmico em lâminas da madeira de Amescla (Trattinnickia burseraefolia), para posterior produção de painéis compensados com o adesivo fenol-formaldeído na gramatura de $200 \mathrm{~g} / \mathrm{m}^{3}$ (linha simples), observaram que os painéis foram classificados como moderadamente resistente à resistente, conforme especificações da norma ASTM D2017 (2005), aos fungos Trametes versicolor e Gloeophyllum trabeum.

Ainda na análise da Tabela 3, verifica-se que a perda de massa foi superior para podridão parda em todos os tratamentos. Estes resultados são devidos a utilização de madeira de conífera (P. taeda) para produção dos painéis, pois, fungos de podridão branca se desenvolvem melhor em madeira de folhosas, enquanto fungos de podridão parda causam maiores perdas de massa em coníferas (CURLING \& MURPHY, 2002; MARTÍNEZ et al., 2005). Apesar de que, quando analisado os painéis após tratamento preservante, tenha sido observada menor perda de massa causada pelo fungo de podridão parda.

Para fins de indicação de uso, quando um material ensaiado apresenta classe de resistência diferente para os fungos testados, por critério de segurança, deve-se considerar os resultados do fungo que apresentou a maior perda de massa. Portanto, apenas os painéis tratados com produtos preservantes podem ser utilizados em situações onde exista risco de apodrecimento. Enquanto que os painéis sem tratamento, que foram classificados como moderadamente resistentes ao ataque do fungo G. trabeum, só devem ser usados fora do contato com a umidade (DIROL e DEGLISE, 2001).

\section{CONCLUSÃO}

Os painéis compensados de Pinus taeda sem tratamento preservativo foram classificados como resistentes a biodeterioração ao fungo Gloeophyllum trabeum e moderadamente resistente ao fungo Trametes versicolor.

Os painéis tratados com os produtos CCA e CCB, nas duas retenções $\left(4,0\right.$ e $\left.6,5 \mathrm{~kg} / \mathrm{m}^{3}\right)$, foram classificados como altamente resistentes, mesmo assim ocorreu melhora significativa de resultados com o aumento da retenção para $6,5 \mathrm{~kg} / \mathrm{m}^{3}$.

$\mathrm{Na}$ avaliação da resistência a biodeterioração dos painéis ao ataque do fungo Trametes versicolor não foi observado efeito significativo do tipo de produto e da retenção aplicada.

$\mathrm{Na}$ avaliação quanto à resistência ao ataque do fungo Gloeophyllum trabeum, o produto CCA aplicado na retenção de $4,0 \mathrm{~kg} / \mathrm{cm}^{3}$ proporcionou maior resistência a biodeterioração que o produto $\mathrm{CCB}$ na mesma retenção e, na avaliação das duas retenções do produto $\mathrm{CCB}$, observouse que o aumento da retenção para $6,5 \mathrm{~kg} / \mathrm{m}^{3}$ resultou em melhora significativa na resistência dos painéis.

\section{AGRADECIMENTOS}

Os autores expressam seus agradecimentos ao Laboratório de Produtos Florestais (LPF) / Serviço Florestal Brasileiro, em Brasília, DF, área de Biodegradação e Preservação da Madeira.

\section{REFERÊNCIAS}

AMARANTE, N.A.; MENDES, L.M.; OKINO, E.Y.A.; GARLET, A.; MORI; MENDES, R.F. Biodegradação de produtos à base da madeira de Cedro australiano (Toona ciliata M. Roem. var. australis). Cerne, Lavras, v. 18, n. 1, p. 17-26, jan./mar. 2012.

\section{AMERICAN SOCIETY FOR TESTING AND}

MATERIALS - ASTM D-2017. Standard method for accelerated laboratory test of natural decay resistance

Cerne, Lavras, v. 20, n. 1, p. 105-112, jan./mar. 2014 
of woods. Annual Book of ASTM Standards, Philadelphia, v.0410, p.5, 2005.

BULCKE, J. VAN DEN; ACKER, J. VAN; SMET, J. DE. An experimental set-up for real-time continuous moisture measurements of plywood exposed to outdoor climate. Building and Environment, v. 44, n. 12, p. 2368-2377, 2009.

CURLING, S. F.; MURPHY, R. J. The use of the Decay Susceptibility Index (DSI) in the valuation of biological durability tests of wood based board materials. Holz als Roh und Werkstoff, n. 60, p. 224-226, 2002.

DIROL, D. \& DEGLISE, X. Durabilitè dês bois et problèmes associés. Hermes Science Europe Ltd. Paris, 2001. 416 p.

FOJUTOWSKI, A.; KROPACZ, A. The susceptibility of hardwood plywood to white rot. Doc. $N^{\circ}$ IRG/WP 08-40397, pg 7, 2008.

GALVÃO, A.P.M.; MAGALHÃES, W. L.E.; MATTOS, P. P. Processos práticos para preservar a madeira. Embrapa Floresta, documento 96, 49 p.,Colombo-PR, 2004.

LOH, Y.; PARIDAH, T.M.; HOONG, Y.B.; BAKAR, E.S.; ANIS, M.; HAMDAN, H. Resistance of phenolic-treated oil palm stem plywood against subterranean termites and white rot decay. International Biodeterioration \& Biodegradation, V. 65, pg.14 -17, 2011.

MARTÍNEZ, A.T.; SPERANZA, M.; RUIZ-DUENAS; FERREIRA, P.; CAMARERO, S.; GUILLÉN F.; MARTÍNEZ, M.J.; GUTIÉRREZ, A.; DEL RIO, J.C. Biodegradation of lignocellulosics: microbial, chimical, and enzymatic aspects of the fungal attack of lignin. International Microbiology, n. 8, p. 195-204. 2005.

MAZELA, B. Changes of copper and chromium content after leaching in Wood impregnated with the CCB and CB preservatives. The International Research
Group on Wood Preservation, 2000. 15p. (IRG/WP, 0050147).

VAN ACKER, J.; STEVENS, M. Effect of various preservative treatments on the mechanical and physical properties of plywood. Doc. N ${ }^{\circ}$ IRG/WP 9340007, pg 3, 1993.

VAZ, S.S. Resistência biológica de painéis compensados de Trattinnickia burseraefolia (mart.) willd. produzidos com lâminas modificadas termomecanicamente. 2013. 29 p. Monografia (Departamento de Engenharia Florestal) - Universidade Federal de Brasília, Brasília.

TASCIOGLU, C., TSUNODA, K., Biological performance of copper azole-treated wood and wood based composites. Holzforschung. V. 64, n.3, p. 39406, 2010a.

TASCIOGLU, C.; TSUNODA, K. Laboratory evaluation of wood-based composites treated with alkaline copper quat against fungal and termite attacks. International Biodeterioration \& Biodegradation, v. 64, n. 8, p. 683-687, 2010b.

TSUNODA, K. Preservative properties of vapor-borontreated wood and wood-based composites. Journal of Wood Science. V. 47, pg. 149-153, 2001.

Recebido: 04 de dezembro de 2012; aceito: 14 de agosto de 2013.

Cerne, Lavras, v. 20, n. 1, p. 105-112, jan./mar. 2014 
